\title{
MONOMORPHISMS, EPIMORPHISMS, AND PULL-BAGKS
}

\author{
G. M. KELLY \\ (Received 7 February 1967; revised 14 August 1967)
}

\section{Introduction}

As the applications of category theory increase, we find ourselves wanting to imitate in general categories much that was at first done only in abelian categories. In particular it becomes necessary to deal with epimorphisms and monomorphisms, with various canonical factorizations of arbitrary morphisms, and with the relations of these things to such limit operations as equalizers and pull-backs.

Call an epimorphism $f: A \rightarrow B$ regular if it is a coequalizer in some general sense (made precise below) and call it extremal if it factors through no proper subobject of $B$. These classes of epimorphisms have been considered by various authors, but most completely by Isbell ([4], [5]). He supposes that the category $\mathscr{A}$ in which we operate satisfies some fairly strong completeness conditions, and then shows the following:

For an arbitrary morphism $f$ there is a smallest regular epimorphism $r$ through which $f$ factors as $f=n r ; r=1$ only when $f$ is monomorphic. In general $n$ is not monomorphic and admits a similar factorization $n=n_{1} r_{1}$; proceeding thus we get $f=n_{\alpha} s_{\alpha}$ where $s_{\alpha}=r_{\alpha} r_{\alpha-1} \cdots r_{1} r$. We can in fact continue transfinitely, and ultimately $n_{\alpha}$ is a monomorphism and the process stops. Then $f=n_{\alpha} s_{\alpha}$ is the unique factorization of $f$ with $n_{\alpha}$ monomorphic and $s_{\alpha}$ an extremal epimorphism. This factorization has good properties, and extremal epimorphisms themselves have good properties, always supposing the original completeness hypotheses to hold; in more general cases extremal epimorphisms are not, for instance, even closed under composition.

The main object of this paper is to consider the relation of these things to pull-backs and push-outs, and in particular to consider the following classes of epimorphisms: we call an epimorphism $f$ persistent if every pullback of $f$ exists and is an epimorphism; very regular if it is persistent and regular; and totally regular if every pull-back of $f$ exists and is itself a regular epimorphism. These are defined (with different names) by Grothendieck ([2], [3]), but there seems to be scarcely anything proved about them in the 
literature. The only results I know of are due to Pupier ([7], Théorème 5) and Oort ([6]); these are in one way or another special, and relate primarily to additive categories; they will be special cases of results proved below.

We try to avoid unnecessary completeness hypotheses and in particular are unwilling to accept those under which, because of the particular applications he has in mind, Isbell establishes his results. It seemed best, therefore, to include an account of regular and extremal epimorphisms, making this in part a survey paper. Our own results appear in section 5, while those in sections 2-4 are essentially Isbell's, re-proved under other and usually weaker conditions. In particular we have abandoned extremal epimorphisms as a basic concept, replacing them by what we call strong epimorphisms. These are always well behaved, and reduce to extremal epimorphisms under mild completeness conditions.

Nomenclature. We use limit and colimit for what some call 'left limit' and 'right limit'. (The dual of any concept is denoted by 'co-' except that the duals of monomorphism, subobject, and pull-back are epimorphism, quotient object, and push-out.) A category is complete [finitely complete] if it admits all small limits [finite limits].

We understand product and fibred product in the usual sense; the fibred product of a family $f_{\alpha}: A_{\alpha} \rightarrow B$ is a family $g_{\alpha}: C \rightarrow A_{\alpha}$. When we take the fibred product of just two morphisms $f_{1}$ and $f_{2}$, we call

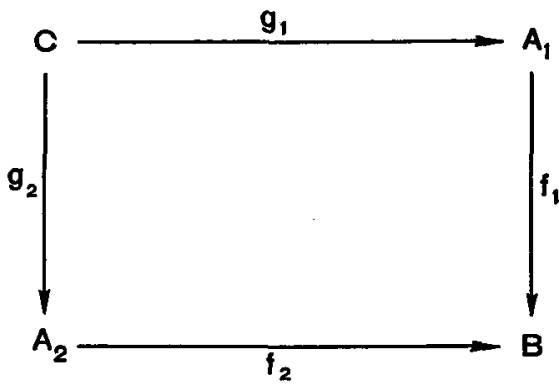

a pull-back diagram, and we say that $g_{2}$ is the pull-back of $f_{1}$ by $f_{2}$. If $A_{1}=A_{2}$ and $f_{1}=f_{2}=f$, we call the pair $g_{1}, g_{2}$ the discriminant of $f$; it is terminal among pairs $x, y$ with $f x=f y$, and has the property that $f$ is monomorphic if and only if $g_{1}=g_{2}$ (in which case we may take $g_{1}=g_{2}=1$ ).

We use the term equalizer only for the equalizer of a single pair $f, g: A \rightarrow B$. By the simultaneous equalizer of a family of pairs $f_{\alpha}, g_{\alpha}: A \rightarrow B_{\alpha}$ we mean a morphism $k: K \rightarrow A$ terminal among those for which $f_{\alpha} k=g_{\alpha} k$ for all $\alpha$.

We call $f$ a monomorphism if $f x=f y$ implies $x=y$. We pre-order monomorphisms with range $A$ by setting $f>g$ if $g$ is of the form $t h$; the 
equivalence classes for the relation ' $f>g$ and $g>f$ ' are called subobjects of $A$. Note that for epimorphisms with domain $A$ we write $f>g$ if $g$ is of the form $h f$. We often confuse a subobject with a representative monomorphism $f$, and we call the subobject regular etc. if the monomorphism $f$ is regular etc. If a family $f_{\alpha}: K_{\alpha} \rightarrow A$ of monomorphisms has fibred product $g_{\alpha}: I \rightarrow K_{\alpha}$, the common value $f_{\alpha} g_{\alpha}$ is also a monomorphism and we call it the intersection of the $f_{\alpha}$, although the term more properly applies to the corresponding subobjects of $A$.

We call the category $\mathscr{A}$ additive if each $\mathscr{A}(A B)$ is an abelian group and composition is bilinear; we do not require finite products to exist.

\section{Regular epimorphisms}

We call a morphism $f: A \rightarrow B$ in a category $\mathscr{A}$ a regular epimorphism if any $g: A \rightarrow C$ satisfying

$$
g x=g y \text { whenever } f x=f y
$$

is of the form $g=h f$ for a unique $h$. The requirement that $h$ be unique may of course be replaced by the equivalent requirement that $f$ be epimorphic.

In other words, $f$ is a regular epimorphism if and only if it is the simultaneous coequalizer of the family of all pairs $x, y$ with $f x=f y$. On the other hand, the simultaneous coequalizer of any family of pairs is seen at once to be a regular epimorphism. In particular the coequalizer of a single pair is a regular epimorphism. A retraction $f$ (that is, a morphism $f$ with $f i=1$ for some $i$ ) is the coequalizer of the pair if, 1 ; and thus is a regular epimorphism. If $\mathscr{A}$ admits discriminants, every regular epimorphism is a coequalizer, namely of its discriminant. If $\mathscr{A}$ is additive every cokernel is a regular epimorphism, since the cokernel of $x$ is the coequalizer of the pair $x, 0$; if further $\mathscr{A}$ admits kernels, every regular epimorphism is a cokernel, namely of its kernel.

Epimorphisms are of course closed under composition, as are retractions. Regular epimorphisms in general are not, even if $\mathscr{A}$ is complete and cocomplete, well-powered and co-well-powered, and additive. Consider the following category $\mathscr{I}$ introduced by Isbell.

$\mathscr{G}$ is the category of abelian groups and $\mathscr{I}$ the full subcategory determined by those groups with no elements of order 4, i.e. those in which $4 x=0$ implies $2 x=0$. For $G$ in $\mathscr{G}$ let $G_{0}$ be the 2-primary subgroup of $G$, let $\Gamma G=G / 2 G_{0}$, and let $\gamma: G \rightarrow \Gamma G$ be the canonical map. Then $\Gamma$ is a reflexion of $\mathscr{G}$ into $\mathscr{I}$, so that $\mathscr{I}$ like $\mathscr{G}$ is complete and cocomplete; limits in $\mathscr{I}$ are formed as in $\mathscr{G}$, and colimits by first forming the colimit in $\mathscr{G}$ and then composing with $\gamma$. It follows too that $\mathscr{I}$ like $\mathscr{G}$ is well-powered; it is easy to see that it is also co-well-powered, for $f: A \rightarrow B$ is epimorphic in 
$\mathscr{I}$ exactly when its cokernel in $\mathscr{I}, \Gamma(B / f A)$, is 0 , i.e. when $B / f A$ is a divisible 2-group, which is impossible if $B$ has no elements of order 4 and if the cardinal of $B$ is infinite and greater than the cardinal of $A$.

The monomorphisms $f: A \rightarrow B$ in $\mathscr{I}$ are those that are monomorphisms in $\mathscr{G}$, and $f$ is a regular monomorphism if and only if it is the kernel of its cokernel in $\mathscr{I}$, i.e. if and only if $B / f A$ is in $\mathscr{I}$. Thus $2: \boldsymbol{Z} \rightarrow \boldsymbol{Z}$ is a regular monomorphism; however its composite with itself, $4: \boldsymbol{Z} \rightarrow \boldsymbol{Z}$, is not. Note that the regular epimorphisms in $\mathscr{I}$ are those that are already epimorphisms in $\mathscr{G}$, and that these are in fact closed under composition.

We do have however:

PROPOSITION 2.1. $f g$ is a regular epimorphism if $f$ is a regular epimorphism and $g$ is a retraction.

Proof. Let $g i=1$, and suppose that $h x=h y$ whenever $f g x=f g y$. Since $f g 1=f g i g$ we have $h 1=h i g$. Next, $f u=f v$ implies $f g i u=f g i v$ and thus $h i u=h i v$; since $f$ is a regular epimorphism we conclude that $h i=k f$ for some $k$. Then $h=h i g=k f g$, as required.

Again, if $f g$ is an epimorphism or a retraction, so is $f$; we say that these classes are closed under right division. The class of regular epimorphisms is not so in general; in the category $\mathscr{I}$ the regular monomorphism $\left(\begin{array}{l}2 \\ 0\end{array}\right): Z \rightarrow Z \oplus Z_{2}$ is the composite of $\left(\begin{array}{l}2 \\ e\end{array}\right): Z \rightarrow Z \oplus Z_{2}$ (where $e \neq 0$ ) and $\left(\begin{array}{c}10 \\ 00\end{array}\right): Z \oplus \boldsymbol{Z}_{2} \rightarrow \boldsymbol{Z} \oplus \boldsymbol{Z}_{2}$, and the first of these is not a regular monomorphism. We do have however:

Proposition 2.2. If $f g$ is a regular epimorphism and if $g$ is an epimorphism, $f$ is a regular epimorphism.

Proof. Let $h x=h y$ whenever $f x=f y$. Then $h g u=h g v$ whenever $f g u=f g v$, so that $h g=k f g$ for some $k, f g$ being a regular epimorphism. Since $g$ is an epimorphism we have $h=k f$, as required.

Proposition 2.3. Let $g_{\alpha}: A \rightarrow B_{\alpha}$ be regular epimorphisms and let $f_{\alpha}: B_{\alpha} \rightarrow C$ be their fibred coproduct. Then the cointersection $f_{\alpha} g_{\alpha}$ is a regular epimorphism, as are all the $f_{\alpha}$.

Proof. By Proposition 2.2 the $f_{\alpha}$ will be regular epimorphisms if $f_{\alpha} g_{\alpha}$ is. Let $h x=h y$ whenever $f_{\alpha} g_{\alpha} x=f_{\alpha} g_{\alpha} y$. Then $h x=h y$ whenever, for some $\alpha, g_{\alpha} x=g_{\alpha} y$; since $g_{\alpha}$ is a regular epimorphism we conclude that $h=k_{\alpha} g_{\alpha}$ for some $k_{\alpha}$. Since $k_{\alpha} g_{\alpha}$ is independent of $\alpha$, it follows from the definition of fibred coproduct that $k_{\alpha}=w f_{\alpha}$ for some $w$. Thus $h=w f_{\alpha} g_{\alpha}$, as required.

REMARK 2.4. Retractions are not closed under cointersections; a counter-example to the dual is given by the following pullback diagram in the category of abelian groups, where $e \neq 0$ : 


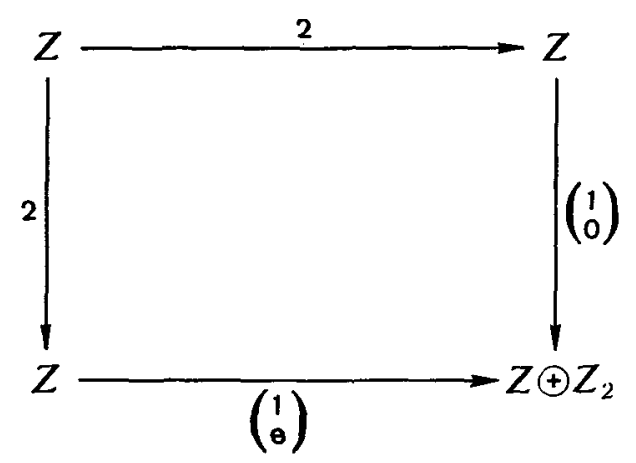

If a morphism $f$ in $\mathscr{A}$ has a factorization $f=n r$ where $r$ is a regular epimorphism and where $r x=r y$ whenever $f x=f y$, we call $n r$ a regular factorization of $f$. It comes to the same thing to say that $r$ is the simultaneous coequalizer of the family of all pairs $x, y$ with $f x=f y$. Thus if a regular factorization of $f$ exists, it is essentially unique. If every $f$ in $\mathscr{A}$ has a regular factorization, we say that $\mathscr{A}$ admits regular factorizations. We shall give in section 4 sufficient conditions for this to be so; it is really in itself a 'completeness condition' of a fairly mild kind.

The following proposition is evident:

Proposition 2.5. If t has the regular factorization $n r, n$ is an isomorphism if and only if $f$ is a regular epimorphism, and $r$ is an isomorphism if and only if $f$ is a monomorphism.

Proposition 2.6. Suppose that $b f=g a$ and that $f, g$ have regular factorizations $f=n r, g=m s$. Then there is a unique $c$ rendering commutative the diagram

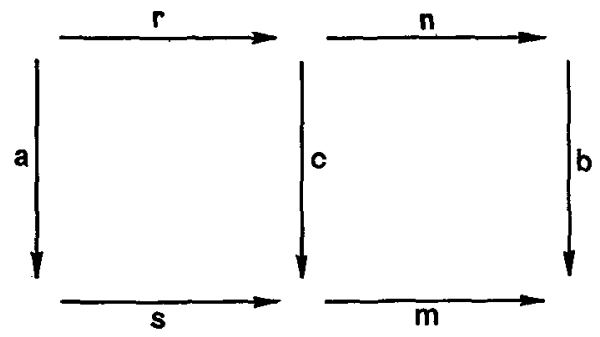

Proof. Whenever $r x=r y$ we have $b n r x=b n r y$ and thus $m s a x=m s a y$. Since $m s$ is a regular factorization we further have sax $=$ say. Since $r$ is a regular epimorphism we conclude that $s a=c r$ for a unique $c$. Then since $b n r=m c r$ and since $r$ is an epimorphism we also have $b n=m c$.

A composite $n r$ is certainly a regular factorization if $r$ is a regular epimorphism and $n$ is a monomorphism. However the $n$ in a regular factorization $n r$ is not in general a monomorphism. In fact: 
Proposition 2.7. If $\mathscr{A}$ admits regular factorizations, the following assertions are equivalent:

(a) Regular epimorphisms are closed under composition.

(b) In every regular factorization $n r, n$ is a monomorphism.

Proof. Given (a) let $f=n r$ be a regular factorization and let the regular factorization of $n$ be $n=m s$. Then $f=m t$, where $t=s r$ is a regular epimorphism by (a) and where $f x=f y$ implies $r x=r y$ and so $t x=t y$; thus $m t$ is a second regular factorization of $f$. The uniqueness of regular factorizations implies that $s$ is an isomorphism and so, by Proposition 2.5, that $n$ is a monomorphism.

Given (b) let $f$ and $g$ be regular epimorphisms and let $f g$ have regular factorization $f g=n r$. Since $l g$ is a regular factorization there is by Proposition 2.6 an $s$ rendering commutative

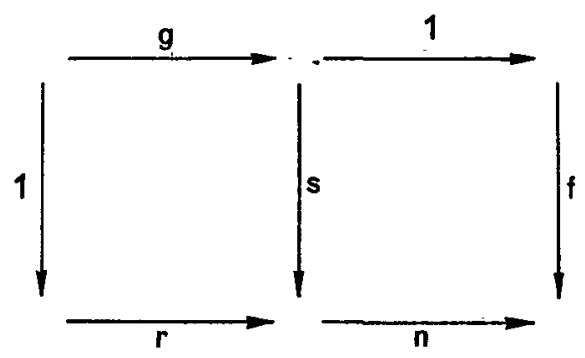

Since $g$ is an epimorphism and since $s g=r$ is a regular epimorphism, $s$ is a regular epimorphism by Proposition 2.2. Since by (b) $n$ is a monomorphism, $n s$ is a regular factorization; but $f=n s$ also has the regular factorization 1 ; we conclude from the uniqueness of regular factorizations that $n$ is an isomorphism and thus that $f g=n r$ is, like $r$, a regular epimorphism.

There is a dual concept of regular cofactorization $f=i m$ where $i$ is a regular monomorphism and where $x f=y f$ implies $x i=y i$. If $f$ has both a regular factorization $n r$ and a regular cofactorization $i m$, Proposition 2.6 applied to the regular factorizations $n r$ and $i 1$, with $1 \cdot n r=i 1 \cdot m$, gives a unique $c$ with $c r=m$ and $i c=n$. We call $f=i c r$ the regular bifactorization of $f$.

\section{Strong epimorphisms}

We call an epimorphism $f$ a strong epimorphism if, whenever $v f=i u$ with $i$ monomorphic, there is a w rendering commutative the diagram 


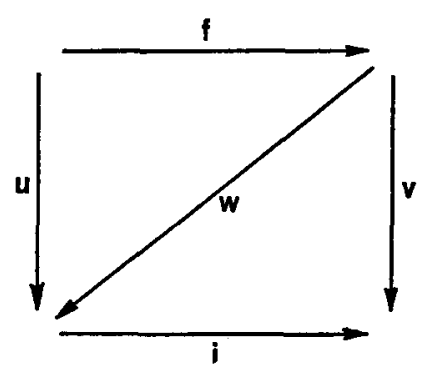

Note that $w$ is unique, and that if either of the tiangles in the above diagram commutes, the other necessarily commutes too, given that $i$ is monomorphic and $f$ epimorphic.

Proposition 3.1. Regular epimorphisms are strong epimorphisms.

Proof. Let $f$ be a regular epimorphism and let $v f=i u$ where $i$ is monomorphic. Applying Proposition 2.6 to the regular factorizations $1 f$ and $i 1$, with $v \cdot 1 f=i 1 \cdot u$, we get a unique $w$ with $v=i w$ and $w=u$.

Proposition 3.2. Strong epimorphisms are closed under composition, right division, and cointersection.

Proof. Composition. Let $v f g=i u$ where $i$ is monomorphic and $f, g$ are strong epimorphisms. Because $g$ is a strong epimorphism there is a $w$ with $i w=v f$. Then because $f$ is a strong epimorphism there is a $z$ with $i z=v$. Thus $f g$ is a strong epimorphism.

RIGHT DIvision. Let $f g$ be a strong epimorphism, and let $v f=i u$ where $i$ is monomorphic. Then $v f g=i$ ing, so that, because $f g$ is a strong epimorphism, there is a $w$ with $i w=v$. Thus $f$ is a strong epimorphism.

Cointersection. Let $g_{\alpha}: A \rightarrow B_{\alpha}$ be strong epimorphisms and let $f_{\alpha}: B_{\alpha} \rightarrow C$ be their fibred coproduct. Let $v f_{\alpha} g_{\alpha}=i u$ where $i$ is monomorphic. Since $g_{\alpha}$ is a strong epimorphism there is a $k_{\alpha}$ with $k_{\alpha} g_{\alpha}=u$. Since $k_{\alpha} g_{\alpha}$ is independent of $\alpha$, there is by the definition of fibred coproduct a $w$ with $k_{\alpha}=w f_{\alpha}$. Then $w f_{\alpha} g_{\alpha}=u$, so that $f_{\alpha} g_{\alpha}$ is a strong epimorphism.

PROPOSITION 3.3. If $f$ is both a strong epimorphism and a monomorphism it is an isomorphism.

Proof. We have $1 f=f 1$ where $f$ is a strong epimorphism and where $f$ is a monomorphism; thus there is a $w$ with $w f=1$ and $f w=1$.

If a morphism $f$ in $\mathscr{A}$ has a factorization $f=i p$ where $p$ is a strong epimorphism and where $i$ is a monomorphism, we call ip a canonical factorization of $f$.

Proposition 3.4. Suppose that $b f=g a$ and that $f, g$ have canonical 
factorizations $f=i p, g=j q$. Then there is a unique $c$ rendering commutative the diagram

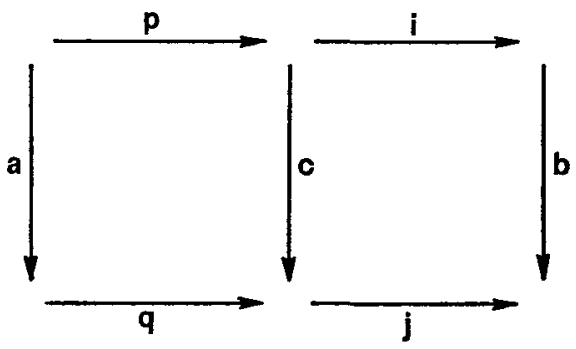

Proof. Apply the definition of strong epimorphism to the equality $b i \cdot p=j \cdot q a$.

\section{COROLLARY 3.5. Canonical factorizations are essentially unique.}

Proof. If in the above proposition $a=1$ and $b=1$, then $c$ is a strong epimorphism because $c p=q$ is, and $c$ is a monomorphism because $j c=i$ is; thus $c$ is an isomorphism by Proposition 3.3.

If $f: A \rightarrow B$ has a canonical factorization $f=i p$, we call the subobject $i$ of $B$ the image of $f$ and the strong quotient object $p$ of $A$ the strong coimage of $f$. The image $i$ is the smallest subobject of $B$ through which $f$ factors; for if $f=t g$ with $t$ monomorphic, we have only to apply Proposition 3.4 to the canonical factorizations ip and $t 1$, with $1 \cdot i p=t / 1 \cdot g$, to see that $i<t$. Similarly the strong coimage $p$ is the smallest strong quotient object of $A$ through which $f$ factors. If $f$ has a factorization $f=j q$ where $j$ is a strong monomorphism and $q$ is an epimorphism, we call $j q$ the canonical cofactorization of $f$, and we call $j$ and $q$ the strong image and the coimage of $f$. If $f$ factorizes as $f=j k p$ where $j$ is a strong monomorphism, $p$ is a strong epimorphism, and $k$ is a bimorphism (i.e. $k$ is both a monomorphism and an epimorphism), we call $j k p$ the canonical bifactorization of $f$. In this case $(j k) p$ is clearly the canonical factorization of $f$, and $j(k p)$ the canonical cofactorization. Conversely, if $f$ has both a canonical factorization ip and a canonical cofactorization $j q$, Proposition 3.4 applied to the canonical factorizations ip and $j 1$ shows the existence of a unique $k$, necessarily a bimorphism, with $f=j k p$.

It follows from the above that a strong epimorphism $f: A \rightarrow B$ factors through no proper subobject of $B$, i.e. that $f$ is an extremal epimorphism.

Proposition 3.6. Extremal epimorphisms are strong epimorphisms if $\mathscr{A}$ admits pull-backs or if $\mathscr{A}$ admits canonical factorizations.

Proof. If $\mathscr{A}$ admits canonical factorizations and if an extremal epimorphism $f$ has canonical factorization $i p, i$ is an isomorphism and so $f$. 
like $p$, is a strong epimorphism. If $\mathscr{A}$ admits pull-backs let $v f=i u$ where $i$ is a monomorphism and $f$ an extremal epimorphism. Form a pull-back diagram

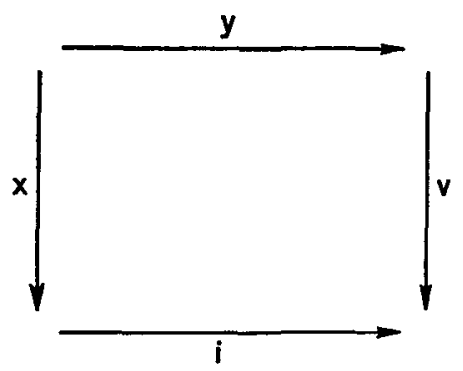

Since $v f=i u$ there is a $z$ with $y z=f$ and $x z=u$. Now it is easy to see that $y$, being the pull-back of a monomorphism $i$, is itself a monomorphism (see Proposition 5.2 below); it is therefore an isomorphism because $f$ is extremal. We now have $v=i x y^{-1}$, so that $f$ is a strong epimorphism.

Without some conditions, extremal epimorphisms are not closed under composition or cointersection, which is why we have considered strong epimorphisms in their stead. There is one consideration however where extremal epimorphisms rather than strong ones arise. It is usual to call a category balanced if every bimorphism is an isomorphism; and it is clear that this is equivalent to the assertion that all epimorphisms are extremal epimorphisms, or equally to the assertion that all monomorphisms are extremal monomorphisms. If we call a category strong when all epimorphisms are strong epimorphisms, strong categories are balanced and the converse is true under mild conditions. This property is self-dual:

Proposition 3.7. If every epimorphism in $\mathscr{A}$ is a strong epimorphism then any monomorphism is a strong monomorphism.

Proof. Dualizing the definition of strong epimorphism, we see that a monomorphism $i$ is strong if, whenever $v f=i u$ with $f$ epimorphic, there is a $w$ with $i w=v$ and $w f=u$. If all epimorphisms $f$ are strong, this is satisfied for any monomorphism $i$, by the definition of strong epimorphism.

PROPOSITION 3.8. If every strong epimorphism in $\mathscr{A}$ is a regular epimorphism, regular epimorphisms are closed under composition. The converse is true provided that $\mathscr{A}$ admits regular factorizations.

Proof. The first assertion is immediate from Proposition 3.1 and Proposition 3.2. For the second, let $f$ be a strong epimorphism and let $f=n r$ be its regular factorization. By Proposition 2.7, $n$ is a monomorphism; since $f$ is an extremal epimorphism, $n$ is an isomorphism; hence $f$ like $r$ is a regular epimorphism. 
We call $\mathscr{A}$ regular if every strong epimorphism is regular, with coregular for the dual; thus the category $\mathscr{I}$ is regular but not coregular. The categories of sets, pointed sets, groups, and compact hausdorff spaces, are strong, regular and coregular. The categories of topological spaces, hausdorff spaces, and short exact sequences of abelian groups, are regular and coregular but not strong. The full subcategory of $\mathscr{I}$ determined by the finitely-generated groups in $\mathscr{I}$ is, unlike $\mathscr{I}$ itself, strong, and is, like $\mathscr{I}$, regular but not coregular. Equational categories of algebras are regular but in general not strong or coregular; that the category of semigroups is not coregular follows from Example 5.I of [5]. We shall see below (immediately before Proposition 5.8) that the category of small categories is not regular.

In a regular category any canonical factorization ip is at the same time a regular factorization. If the category admits regular factorizations, any regular factorization $n r$ is also a canonical factorization, for $n$ is monomorphic by Propositions 3.8 and 2.7. Thus if $\mathscr{A}$ admits either type of factorization it admits the other and they coincide. In more general categories the relation between the two kinds of factorization is shown by the following proposition:

Proposition 3.9. Let $\mathscr{A}$ admit regular factorizations and cointersections. Given a morphism $f: A \rightarrow B$ define for each ordinal $\alpha$ a factorization $f=n_{\alpha} \nu_{\alpha}$ by the following transfinite induction:

(i) let $r_{0}=1$ and $n_{0}=t$;

(ii) if $\alpha=\beta+1$, let $n_{\alpha} s_{\alpha}$ be the regular factorization of $n_{\beta}$ and set $r_{\alpha}=s_{\alpha} r_{\beta}$

(iii) if $\alpha$ is a limit ordinal, let $r_{\alpha}$ be the cointersection of the $r_{\beta}$ with $\beta<\alpha$.

Then if $r_{\alpha}$ is stationary for $\alpha \geqq$ some $\lambda$, which it will be if $A$ has only a set of strong quotient objects, $f=n_{\lambda} r_{\lambda}$ is the canonical factorization of $f$.

PROOF. Each $r_{\alpha}$ is a strong epimorphism by Propositions 3.1 and 3.2. By Proposition 2.5 we have $r_{\lambda+1}=r_{\lambda}$ if and only if $n_{\lambda}$ is a monomorphism, which means that we have arrived at the canonical factorization of $f$.

\section{Existence of factorizations}

LeMma 4.1. If $f: A \rightarrow B$ has a regular factorization $f=n r, r$ is the smallest regular quotient object of $A$ through which $f$ factors. Conversely if there is a smallest regular quotient object $r$ of $A$ through which $f$ factors, and if further $\mathscr{A}$ admits coequalizers, $f$ has a regular factorization $f=n r$.

Proof. For the first statement, let $f=m s$ where $s$ is a regular epimorphism. Proposition 2.6 applied to the regular factorizations $1 s$ and $n r$ shows that $r<s$. For the second statement let $n r$ be the factorization of $f$ 
through $r$ and let $f x=f y$. If $s$ is the coequalizer of $x, y$ then $f$ factors through the regular epimorphism $s$ and so $r<s$; since $s x=s y$ we have $r x=r y$, as desired.

Weak limits are defined exactly like limits, except that the uniqueness requirement is dropped from the definition. Thus a weak discriminant $u, v$ of $f$ is a pair such that $f u=f v$ and such that whenever $f x=f y$ we have $x=u k, y=v k$ for some $k$.

PROPOSITION 4.2. $\mathscr{A}$ admits regular factorizations if either of the following assertions is true:

(a) $\mathscr{A}$ admits coequalizers and weak discriminants.

(b) $\mathscr{A}$ admits coequalizers and cointersections, and every object has only a set of regular quotient objects.

Proof. Let $f: A \rightarrow B$ be a morphism. In case (a) let $u, v$ be a weak discriminant of $f$ and let $\boldsymbol{r}$ be the coequalizer of $u, v$; clearly $f$ has a regular factorization $n r$. In case (b) the cointersection of those regular subobjects of $A$ through which $f$ factors is by Proposition 2.3 the smallest such, and we appeal to Lemma 4.1.

LEMMA 4.3. The following assertions are equivalent:

(a) $\mathscr{A}$ admits canonical factorizations.

(b) Given $f: A \rightarrow B$ there is a smallest subobject of $B$ through which $f$ factors; and if $f$ factors through no proper subobject of $B$ it is a strong epimorphism.

(c) Given $f: A \rightarrow B$ there is a smallest strong quotient object of $A$ through which $f$ factors; and if $f$ factors through no proper strong quotient object of $A$, it is a monomorphism.

Proof. This is evident in view of the remarks about images and strong coimages in section 3.

Lemma 4.4. If $\mathscr{A}$ admits equalizers, a morphism $f: A \rightarrow B$ that factors through no proper strong subobject of $B$ is an epimorphism.

Proof. If $x f=y f, f$ factorizes through the equalizer of $x, y$, which is a strong subobject of $B$. Therefore this equalizer is an isomorphism, and $x=y$.

Proposition 4.5. $\mathscr{A}$ admits canonical factorizations if either of the following assertions is true:

(a) $\mathscr{A}$ admits intersections, equalizers, and pull-backs, and each object has only a set of subobjects.

(b) $\mathscr{A}$ admits cointersections and coequalizers, and each object has only a set of strong quotient objects. 
Proof. In case (a), given $f: A \rightarrow B$, let $i: I \rightarrow B$ be the intersection of all the subobjects of $B$ through which $f$ factors, and so the smallest such. If $f=i p, p$ factors through no proper subobject of $I$; therefore $p$ is an extremal epimorphism by Lemma 4.4 and a strong epimorphism by Proposition 3.6.

In case (b), let $p: A \rightarrow I$ be the cointersection of all the strong quotient objects of $A$ through which $f$ factors, and so the smallest such. If $f=i p$, $i$ factors through no proper strong quotient object of $I$, and thus $i$ is a monomorphism by Lemma 4.4.

The conditions in Propositions 4.2 and 4.5 about subobjects of certain kinds forming a set may be implied by the existence of generating sets of certain kinds. A set $\{G\}$ of objects of $\mathscr{A}$ is called a generating set if, whenever $f \neq g: A \rightarrow B$, there is a $G$ in $\{G\}$ and a $w: G \rightarrow A$ with $f w \neq g w$. If $T$ is the functor from $\mathscr{A}$ to sets given by $T A=\sum_{G} \mathscr{A}(G A)$, it comes to the same thing to say that $T$ is faithful. This implies that $f$ is epimorphic whenever $T f$ is, and similarly for monomorphic. If $f$ is known to be a strong monomorphism, it follows that $f$ is an isomorphism if $T f$ is an isomorphism. The generating set $\{G\}$ is called a strong generating set if $f$ is always an isomorphism when $T f$ is an isomorphism.

Proposition 4.6. If $\mathscr{A}$ has a generating set, each object has only a set of regular quotient objects; if further $\mathscr{A}$ admits finite intersections, each object has only a set of strong subobjects; if besides all this the generating set is strong, each object has only a set of subobjects.

PRoof. If $\{G\}$ is the generating set, a regular epimorphism $r: A \rightarrow C$ is the simultaneous coequalizer of the family of all pairs $x, y: G \rightarrow A$ with $G$ in $\{G\}$ and $r x=r y$; there are only a set of such families.

If $\mathscr{A}$ admits finite intersections define the functor $T$ as above. Note that two subobjects $i, j$ of $A$ are different if and only if, in the pull-back diagram

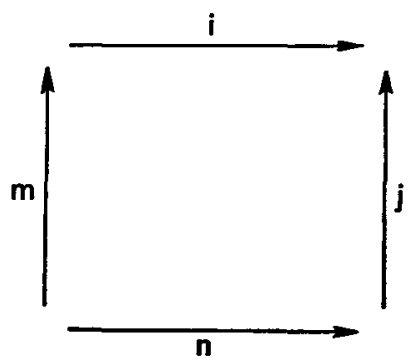

at least one of $m, n$ is a non-isomorphism. Note further that, if $i, j$ are strong monomorphisms, so are $m, n$ by Proposition 3.2. Since $T$ preserves both monomorphisms and intersections, $T$ maps the strong subobjects 
(or if $\{G\}$ is strong, the subobjects) of $A$ injectively into those of $T A$, of which there are only a set.

\section{Relations with pull-backs}

We shall constantly use the following lemma, parts of which at least are well known; the easy proof is left to the reader.

LEMMA 5.1. (a) In the commutative diagram

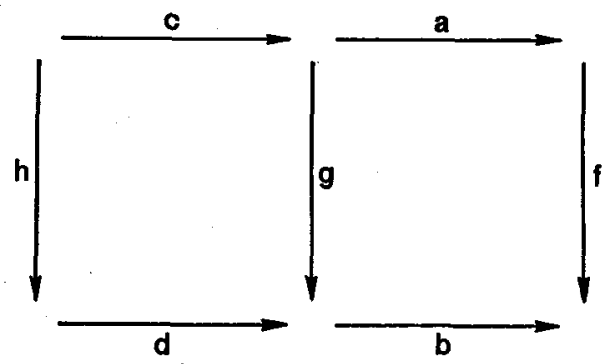

let the right square be a pull-back. Then the left square is a pull-back if and only if the exterior rectangle

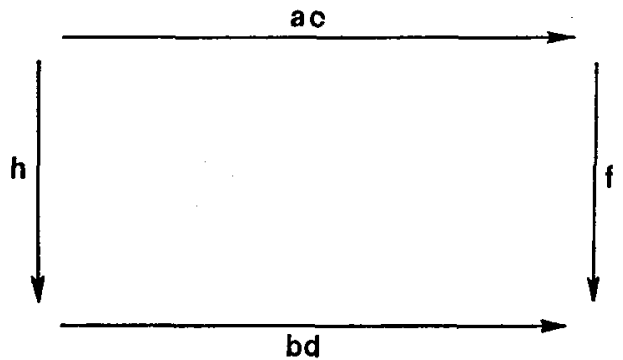

is a pull-back.

(b) If the diagrams
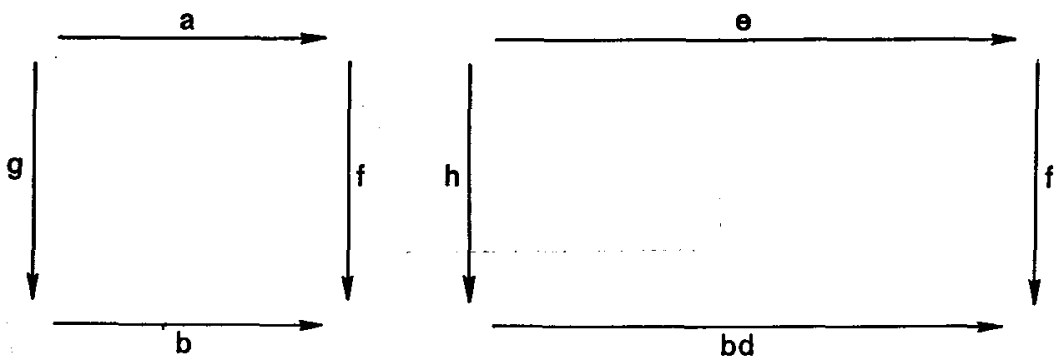

are both pull-backs, there is a unique $c$ with $e=a c$ and $d h=g c$. 
We say that a class of morphisms is preserved under push-outs if, whenever $g$ is of the given class and

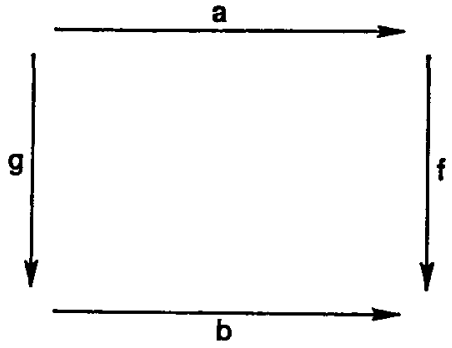

is a push-out diagram, $f$ too is of the given class.

Proposition 5.2. Epimorphisms, strong epimorphisms, and regular epimorphisms are preserved under push-outs.

Proof. Suppose we have the push-out diagram above. If $g$ is an epimorphism let $x f=y f$. Then $x f a=y f a$ and so $x b g=y b g$; thus $x b=y b$ since $g$ is an epimorphism. By the uniqueness clause in the definition of push-out, from $x f=y f$ and $x b=y b$ we deduce $x=y$. Thus $f$ is an epimorphism.

If $g$ is a strong epimorphism let $v f=i u$ where $i$ is monomorphic. Then $v b g=v f a=i u a$, and so since $g$ is a strong epimorphism there is a $z$ with $z g=u a$. Since the diagram is a push-out, there is a $w$ with $u=w f$. Thus $f$ is a strong epimorphism.

If $g$ is a regular epimorphism let $h x=h y$ whenever $f x=f y$. Then $h a w=h a z$ whenever faw $=f a z$, that is whenever $b g w=b g z$, and in particular whenever $g w=g z$. Since $g$ is a regular epimorphism we have $h a=k g$ for some $k$. Since the diagram is a push-out we have $h=q f$ for some $q$. Thus $f$ is a regular epimorphism.

REMARK 5.3. The example of Remark 2.4 shows that retractions are not preserved under push-outs.

Proposition 5.4. If the above diagram is a push-out and if $f$ is epimorphic so is $g$, provided that the category is additive.

Proof. If $x g=0$ then $x g=0 a$, so that for some $y$ we have $x=y b$, $0=y f$. Since $f$ is an epimorphism we have $y=0$ and so $x=0$.

REMARK 5.5. In a pointed category with null-object $O$ the push-out of $f: A \rightarrow B$ by $0: A \rightarrow O$ is the isomorphism $0: O \rightarrow O$ provided that the cokernel of $f$ is 0 ; which does not imply that $f$ is epimorphic. Thus Proposition 5.4 is false in the non-additive case and is best-possible in the additive case. 
We now consider preservation under pull-backs.

Proposition 5.6. Retractions are preserved under pull-backs.

Proof. Let the diagram above be a pull-back and suppose that $f i=1$. Then we also have the pull-back diagram

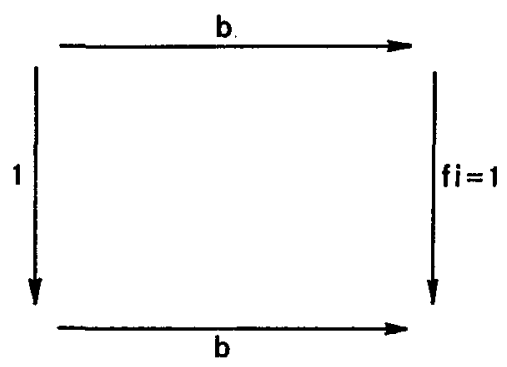

It follows from Lemma 5.1(b) that $\mathrm{I}=g j$ for some $j$.

REMARK 5.7. A pull-back of a regular epimorphism need not even be an epimorphism. The following diagram (where $e \neq 0$ ) is a push-out in the category $\mathscr{I}$ of section 2 , and 2 is a regular monomorphism:

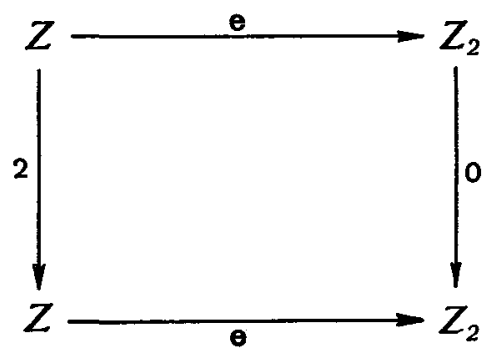

We have given in section 1 the definitions of persistent epimorphism, very regular epimorphism, and totally regular epimorphism. Proposition 5.6 shows that a retraction is totally regular provided all its pull-backs exist. Totally regular epimorphisms need not be retractions; every epimorphism is totally regular in the category of abelian groups. In the category of topological spaces the epimorphisms are the surjections and they are all persistent; the strong and the regular epimorphisms coincide and are the identification maps; thus every identification map is very regular. However they are not all totally regular; Bourbaki ([1], p. 151, ex. 6) gives an example of an identification map $f: X \rightarrow Y$ and a space $Z$ such that

$$
f \times 1: X \times Z \rightarrow Y \times Z
$$

is not an identification map; and $f \times 1$ is the pull-back of $f$ by the projection $Y \times Z \rightarrow Y$. Remark 5.7 shows that regular epimorphisms need not be very 
regular. On the other hand a strong persistent epimorphism, even one all of whose pullbacks are strong epimorphisms, need not be regular, as is shown by the following example kindly provided by the referee. $\mathscr{A}$ is the category of small categories, $A$ the category with two objects $P, Q$ and one non-identity map $s: P \rightarrow Q, B$ the category with one object $R$ and a single non-identity map $t: R \rightarrow R$ satisfying $t^{2}=t$; an epimorphism of the required kind is the functor $f: A \rightarrow B$ taking $s$ to $t$.

From Lemma 5.1 we conclude at once:

Proposition 5.8. Persistent epimorphisms and totally regular epimorphisms are preserved under pull-backs.

We call a diagram

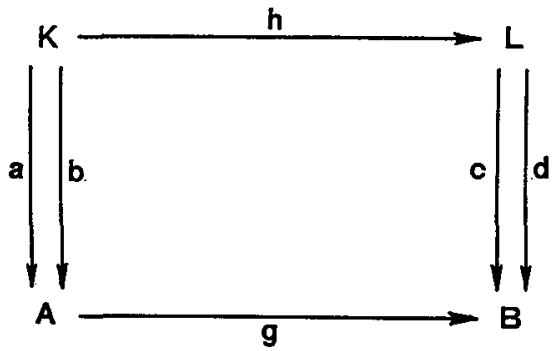

a pull-back of pairs if

(i) $c h=g a$ and $d h=g b$;

(ii) whenever $c z=g x$ and $d z=g y$ we have $x=a w, y=b w, z=h w$ for some unique $z$.

We call $a, b$ the pull-back by $g$ of $c, d$, and we call $h$ the pull-back by $c, d$ of $g$. If finite products exist it comes to the same thing to say that the diagram

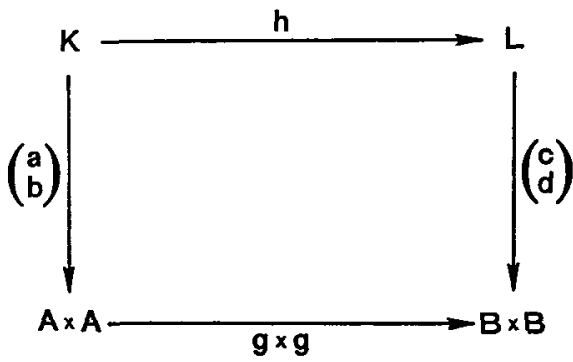

is an ordinary pull-back, but we do not want to assume unnecessarily that products exist.

Lemma 5.9. If all pull-backs of $g$ exist, so do all pull-backs of $g$ by pairs. If further all pull-backs of $g$ are epimorphisms, so are all pull-backs of $g$ by pairs. 
Proof. In the diagram below, let each cell be a pull-back:

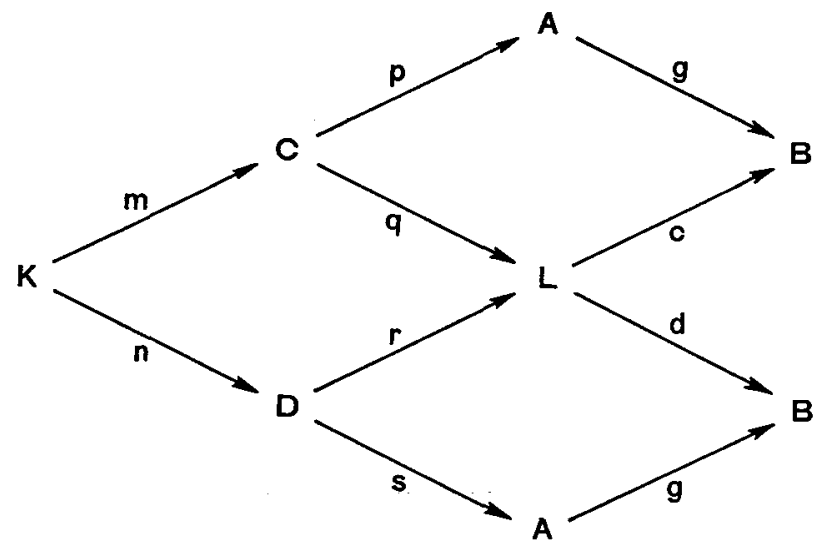

The right cells exist by hypothesis, and the left cell exists by Proposition 5.8. If we set $a=p m, b=s n$, and $h=q m=r n$, it is immediate that we have a pull-back of pairs with the notation as in the above definition. If every pull-back of $g$ is an epimorphism, then $r, n$ are epimorphisms ( $n$ being a pull-back of $g$ by Lemma 5.1), and so $h=r n$ is an epimorphism.

Proposition 5.10. The composite $t g$ is a regular epimorphism if $f$ is a regular epimorphism and $g$ is a very regular epimorphism.

PRoor. Let $k x=k y$ whenever $f g x=f g y$. Then $k x=k y$ whenever $g x=g y$, and so, because $g$ is a regular epimorphism, $k=w g$ for some $w$. Now let $f c=f d$, and form the pull-back of $g$ by the pair $c, d$ with the notation as in the above definition; this exists by Lemma 5.9, and by the same lemma $h$ is an epimorphism. Since $f c=f d$ we have $f c h=f d h$ and thus $f g a=f g b$; by hypothesis therefore we have $k a=k b$, that is $w g a=w g b$, and thus $w c h=w d h$. Since $h$ is an epimorphism, we conclude that $w c=w d$. Because this is true whenever $f c=f d$ and because $f$ is a regular epimorphism, $w=z f$ for some $z$. Thus $k=w g=z f g$, as required.

Proposition 5.11. Persistent, very regular, and totally regular epimorphisms are closed under composition.

Proof. The result for persistent epimorphisms is immediate from Lemma 5.1. From this result together with Proposition 5.10 we get the result for very regular epimorphisms. From this result together with Lemma 5.1 and Proposition 5.8 we get the result for totally regular epimorphisms.

Proposition 5.12. If $\mathrm{fg}$ is a persistent or a very regular or a totally regular epimorphism, so is $f$, provided that all pull-backs of $f$ exist. 
Proof. For persistent epimorphisms this is immediate from Lemma 5.1. Once we have proved it for very regular epimorphisms it follows for totally regular epimorphisms by Lemma 5.1 and Proposition 5.8. Suppose then that $f g$ is a very regular epimorphism; we have to show that $f$ is regular. Form a pull-back diagram

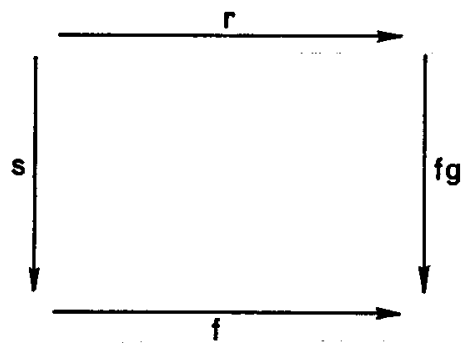

Since $f g \cdot 1=f \cdot g$ there is a $t$ with $1=r t, g=s t$; so $r$ is a retraction. Therefore $f g r$ is a regular epimorphism by Proposition 2.1. Thus $f s$ is a regular epimorphism, and $s$ is an epimorphism because $f g$ is a very regular epimorphism; therefore $f$ is a regular epimorphism by Proposition 2.2.

REMARK 5.13. The above classes of epimorphisms are not in general closed under cointersection or preserved by push-outs; this is shown by the diagram of Remark 2.4, interpreted now however as a pull-back not in $\mathscr{G}$ but in $\mathscr{I}$; we saw in Remark 5.7 that 2 is not a persistent monomorphism in $\mathscr{I}$.

From Proposition 3.8 and Proposition 5.10 we have:

PROPOSITION 5.14. If every regular epimorphism in $\mathscr{A}$ is very regular, then every strong epimorphism in $\mathscr{A}$ is regular (i.e. $\mathscr{A}$ is regular), provided that $\mathscr{A}$ admits regular factorizations.

Remark 5.15. The converse of Proposition 5.14 is false. The category of hausdorff spaces is coregular, since the regular monomorphisms are those $f: A \rightarrow B$ where $A$ is a closed subspace of $B$ and $f$ is the inclusion, and these are closed under composition. Yet not all regular monomorphisms are very regular. Let $X$ be a non-normal hausdorff space and $A, B$ disjoint closed sets in $X$ which do not have disjoint neighbourhoods. Let $C$ be the closed subspace $A \cup B$ of $X$; the inclusion $i: C \rightarrow X$ is a regular monomorphism. Let $D$ be the discrete space consisting of two points $x$ and $y$, and define $f: C \rightarrow D$ by $f(A)=x, f(B)=y$. The push-out of $i$ by $f$ in the category of all topological spaces is the map $g: D \rightarrow E$, where $E$ is obtained from $X$ by identifying $A$ to a single point $a$ and $B$ to a single point $b$, and where $g(x)=a, g(y)=b$. The push-out of $i$ by $f$ in the category of hausdorff spaces is the composite $D \underset{g}{\rightarrow} E \underset{h}{\rightarrow} F$, where $F$ is the greatest hausdorff quotient space of $E$. But $h$ identifies $a$ and $b$, which have not disjoint neighbourhoods in $E$; so that $h g$ is not a monomorphism. 
If $\mathscr{A}$ is additive, however, the converse of Proposition 5.14 is true:

Proposition 5.16. Let $\mathscr{A}$ be additive and finitely complete. If every strong epimorphism is regular then every regular epimorphism is very regular.

Proof. Consider a pull-back diagram

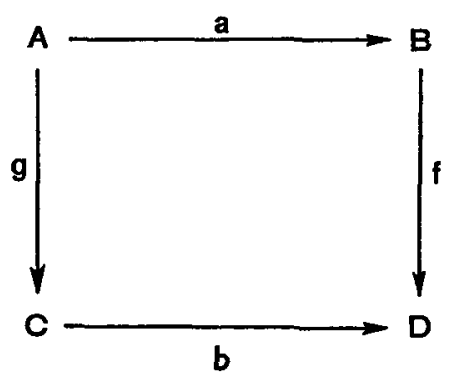

where $f$ is a regular epimorphism. To say that this is a pull-back diagram is to say that $\left(\begin{array}{c}a \\ -g\end{array}\right): A \rightarrow B \oplus C$ is the kernel of $(f b): B \oplus C \rightarrow D$. Since the composite of $(f b)$ with $\left(\begin{array}{l}1 \\ 0\end{array}\right): B \rightarrow B \oplus C$ is the strong epimorphism $f$, it follows from Proposition $\mathbf{3 . 2}$ that $(f b)$ is a strong epimorphism. It is therefore by hypothesis a regular one, and therefore it is the cokernel of its kernel $\left(\begin{array}{c}a \\ -g\end{array}\right)$. This, however, implies that the diagram above is a pushout, and now it follows from Proposition 5.4 that $g$ is an epimorphism, as required.

\section{References}

[1] N. Bourbaki, Eléments de mathématique: Livre III (Topologie générale) (Ch. I, $3^{\mathrm{me}}$ Éd. Paris, 1961).

[2] A. Grothendieck, 'Technique de descente et théorèmes d'existence en géométrie algébrique. I,' Séminaire Bourbaki 12 (1959/60), Exp. 190.

[3] A. Grothendieck, 'Techniques de construction et théorèmes d'existence en géométrie algébrique. III', Séminaire Bourbaki 13 (1960/61), Exp. 212.

[4] J. R. Isbell, 'Subobjects, adequacy, completeness and categories of algebras', Rozprawy Mat. 36 (1964) $1-32$.

[5] J. R. Isbell, 'Structure of categories', Bull. Amer. Math. Soc. 72 (1966), 619-655.

[6] F. Oort, 'On the definition of an abelian category', Nederl. Akad. Wetensch. Proc. Ser. A 70 (Indag. Math. 29) (1967), 83-92.

[7] R. Pupier, 'Sur les décompositions de morphismes dans les catégories à sommes ou à produits fibrés', C. R. Acad. Sci. Paris 258 (1964), 6317-6319.

The University of

New South Wales 